Trauma Berufskrankh 2014 • 16[Suppl 3]:251-253 DOI 10.1007/s10039-014-2060-z

Online publiziert: 19. April 2014

(c) Springer-Verlag Berlin Heidelberg 2014

T. Yilmaz $\cdot$ M. Jaeger $\cdot$ N.P. Südkamp

Klinik für Orthopädie und Unfallchirurgie, Universitätsklinikum Freiburg

\title{
Winkelstabile Platte bei proximaler Humerusfraktur
}

\section{Standard der Versorgung?}

kelstabile Platten, zur Verfügung. Ziele sowohl der konservativen als auch der operativen Versorgung sollten die Herstellung der schmerzfreien Schulterbeweglichkeit und die Einsatzfähigkeit der verletzten Schulter im Alltag sein.

\section{Konservative Therapie}

„Fractures with minimum displacement, regardless of the number of fracture lines, can be satisfactorily treated by early functional exercises" [6].

Diese von Neer postulierte These zur konservativen Behandlung von proximalen Humerusfrakturen sollte nach dem aktuellen Stand der Literatur relativiert werden. Die konservative Behandlung der Humeruskopffrakturen stellt eine gute Therapieoption dar. Mit ihr lassen sich frakturtypabhängig sehr gute Ergebnisse erzielen [1,2]. Zur konservativen Therapie eignen sich unabhängig vom Allgemeinzustand, Patientenalter und -wunsch besonders Frakturen, die nur gering disloziert und/oder intrinsisch stabil sind.

Instabile Frakturen entstehen durch eine metaphysäre Zerreißung des Periostschlauchs oder eine metaphysäre Trümmerzone. Man erkennt sie an einer Dislokation des proximalen Humerusschafts um mehr als 1/3 der Schaftbreite. Sie neigen unter konservativer Therapie zur Ausbildung von Pseudarthrosen, mit einer Häufigkeit von bis zu 10\%. Die jün- gere Literatur zeigt auf Level-1-Evidenz divergierende Resultate: So konnten auch unter konservativer Therapie $z$. T. sehr gute Ergebnisse bei der Behandlung von dislozierten 3- und 4-Segment-Frakturen beobachtet werden. Im direkten Vergleich zur Plattenosteosynthese oder frakturprothetischen Versorgung konnten nach konservativer Behandlung keine signifikanten Nachteile herausgestellt werden. Jedoch machten Olerud et al. [8] darauf aufmerksam, dass der weithin gebräuchliche Constant-Score u. U. nicht geeignet ist, als Endpunkt zur Beurteilung zu dienen. Sie beobachteten eine Revisionsrate von 30\% nach Plattenosteosynthese.

\section{Operative Therapie}

Die Grenzen der konservativen Therapie zeigen sich v. a. bei instabilen und deutlich dislozierten Frakturen. Diese erfordern die offene Reposition und osteosynthetische Versorgung. Zur Osteosynthese stehen Kirschner-Drähte, Marknägel und Platten zur Verfügung. Dabei setzten sich weltweit winkelstabile Systeme durch. Während in der Vergangenheit monoaxiale Kopfverriegelungsschrauben benutzt wurden, stehen nunmehr auch polyaxiale Kopfverriegelungsschrauben sowie zementaugmentierbare Schraubensysteme zur Verfügung. Der klinische Vorteil der letztgenannten Implantate muss jedoch noch durch weitere klinische Studien dokumentiert werden. Die 


\section{Schultergürtel}

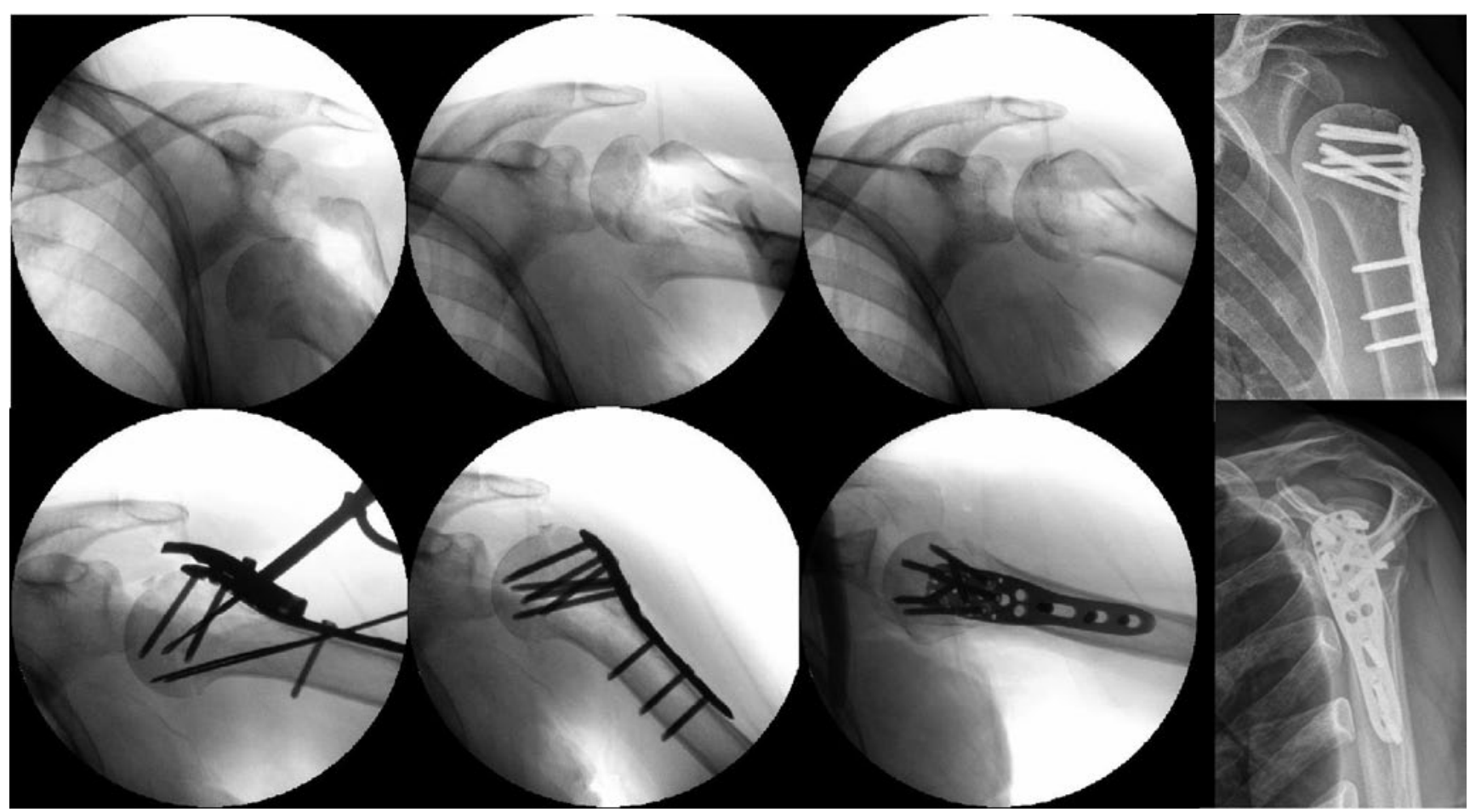

Abb. $1 \Delta$ Behandlung einer 4-Segment-Humeruskopfluxationsfraktur mittels PHILOS („proximal humeral internal locked system"): links intraoperative Bilder: korrekte Reposition und Wiederherstellung (obere Reihe) des Kalkars als Voraussetzung der Fixation mittels PHILOS (untere Reihe), rechts radiologische Verlaufskontrollen (postoperativ)

polyaxialen Schrauben bieten gegenüber den monoaxialen keinen Vorteil [7]. Zur Stabilisierung kommen häufig Marknägel und winkelstabile Platten zum Einsatz.

\section{Humeruskopf-2- und -3-Segment-Frakturen}

Eine wichtige Rolle bei der Implantatwahl spielt neben der bereits oben erwähnten Frakturmorphologie auch die Evidenz der einzelnen Verfahren. Allerdings sind Studien mit einem hohen Evidenzlevel, in denen die einzelnen Verfahren mit ausreichender Patientenzahl verglichen wurden, nur in geringer Anzahl vorhanden.

Die Humeruskopf-2-Segment-Frakturen am chirurgischen Hals zeigten bei ihrer Behandlung mittels Marknageloder winkelstabiler Plattenosteosynthese im klinischen Outcome nach 3 Jahren keine Unterschiede. Solche waren jedoch bei einem Vergleich nach dem ersten Jahr nach der Versorgung feststellbar: Bei den mittels Marknagel versorgten Patienten fanden sich zu diesem Zeitpunkt im Vergleich zu den mittels winkelstabiler Platte versorgten Verletzten geringere postoperative Komplikationsraten. Im Gegenzug war bei Letzteren das klinische Outcome besser [12].

Konrad et al. [4] dokumentierten 1 Jahr nach der Operation ein vergleichbares Ergebnis in der Behandlung von Humeruskopf-3-Segment-Frakturen. Sie werteten 211 Patienten aus, die entweder mit einem proximalen Humerusnagel (PHN) oder mit einer winkelstabilen Platte [PHILOS (,proximal humeral internal locked system“), LPHP („locking proximal humerus plate")] versorgt worden waren. Erstere (PHN) hatten nach 3, 6 und 12 Monaten zwar signifikant weniger Schmerzen, jedoch zeigten die Constant-Score-Werte nach 1 Jahr keine signifikanten Unterschiede [4].

Zusammengefasst werden somit bei einer Versorgung von Humeruskopf-2und -3-Segment-Frakturen mit Nagel bzw. winkelstabiler Platte ähnliche Resultate erzielt.

\section{Winkelstabile Platte als Standardverfahren der Humeruskopf-4-Segment-Fraktur}

Zur Versorgung der Humeruskopf-4Segment-Frakturen bewährten sich winkelstabile Platten [11]. Sie werden bevor- zugt über einen deltoideopektoralen $\mathrm{Zu}$ gang eingebracht.

Neben der korrekten Implantatwahl ist es eminent wichtig, die verschiedenen Einflussfaktoren zu kennen, die an einem schlechten Outcome beteiligt sind. Südkamp et al. [10] entwickelten diesbezüglich eine Pathway-Analyse, mit der sie weibliches Geschlecht, ein Patientenalter von über 40 Jahren, eine Varusfehlstellung von über $30^{\circ}$ und das Auftreten intraoperativer Komplikationen als negative Einflussfaktoren identifizieren konnten.

Krappinger et al. [5] hoben das Patientenalter, die Knochendichte, die anatomische Reposition und die Rekonstruktion des Kalkars besonders hervor. Weist der Patient keinen oder nur einen dieser Risikofaktoren auf, ist die postoperative Komplikationsrate gering. Bei Vorliegen von 2 Risikofaktoren erhöht sich das Risiko merklich auf etwa 9\%. Bei 3 und mehr Risikofaktoren nimmt das portoperatives Komplikationsrisiko drastisch zu und beträgt bei 3 Risikofaktoren $71 \%$ und bei 4 Risikofaktoren 85\% [5].

Während sich das Patientenalter [3] und die Knochendichte [9] zum Zeitpunkt der Operation nicht beeinflussen 
lassen, liegt das Repositionsergebnis allein in der Hand des Operateurs. Sein Geschick ist ausschlaggebend dafür, ob eine anatomische Reposition und die Rekonstruktion des Kalkars und somit die Steigerung der intrinsischen Stabilität der Fraktur möglich sind. Unter Umständen ist eine leichte Impaktion des Schafts geeignet, die Stabilität zu erhöhen. Fibulagrafts oder andere Knochenersatzmaterialien müssen nicht routinemäßig verwendet werden ( $\bullet$ Abb. 1).

\section{Fazit für die Praxis}

- Bei der Versorgung der proximalen Humerusfrakturen haben alle Verfahren, je nach Verletzungsschwere und Patient, ihren Stellenwert.

- Ist eine konservative Therapie nicht möglich, sollte v. a. bei 4-SegmentFrakturen die winkelstabile Platte verwendet werden. Sie stellt aus unserer Sicht das Standardimplantat zur Versorgung der dislozierten 4-SegmentFrakturen dar.

- Deutlich wichtiger als die Implantatauswahl ist die gute und möglichst anatomische Reposition.

\section{Korrespondenzadresse}

\section{Dr. T. Yilmaz}

Klinik für Orthopädie und Unfallchirurgie, Universitätsklinikum Freiburg, Hugstetter Straße 55, 79106 Freiburg tayfun.yilmaz@uniklinik-freiburg.de

\section{Einhaltung ethischer Richtlinien}

Interessenkonflikt. T. Yilmaz, M. Jaeger und N. P. Südkamp geben an, dass kein Interessenkonflikt besteht.

Dieser Beitrag beinhaltet keine Studien an Menschen oder Tieren.

The supplement containing this article is not sponsored by industry.

\section{Literatur}

1. Hauschild O, Konrad G, Audige L et al (2013) Operative versus non-operative treatment for two-part surgical neck fractures of the proximal humerus. Arch Orthop Trauma Surg 133:1385-1393
Trauma Berufskrankh 2014 • 16[Suppl 3]:251-253 DOI 10.1007/s10039-014-2060-z

(c) Springer-Verlag Berlin Heidelberg 2014

T. Yilmaz $\cdot$ M. Jaeger $\cdot$ N.P. Südkamp

Winkelstabile Platte bei proximaler Humerusfraktur. Standard der Versorgung?

\section{Zusammenfassung}

Epidemiologie. Humeruskopffrakturen stellen die dritthäufigsten Frakturen des Menschen dar und betreffen v. a. den älteren Patienten.

Therapiewahl. Zur Versorgung von Humeruskopffrakturen stehen sowohl konservative als auch operative Behandlungsmöglichkeiten zur Verfügung. Die Behandlungsoption sollte abhängig von der Frakturmorphologie und vom Dislokationsgrad gewählt werden. Langzeitergebnisse ließen keine Unterschiede zwischen der Versorgung von 2- und 3-Segment-Frakturen mittels Marknagel und winkelstabiler Platte erkennen. Standard der operativen Versorgung der nichtkonservativ

behandelbaren 4-Segment-Frakturen sollte die winkelstabile Plattenosteosynthese sein. Vermeidung von Komplikationen. Unabhängig vom Verfahren sollten die Risikofaktoren für das Auftreten postoperativer Komplikationen vermieden werden. Daher sind sowohl die anatomische Reposition der Fraktur als auch die Rekonstruktion des Kalkars essenziell.

\section{Schlüsselwörter}

Humeruskopf · Knochennägel .

Knochenplatte - Anatomische Reposition . Rekonstruktion des Kalkars

\section{Locking plate for proximal humeral fractures. Standard of care?}

\section{Abstract}

Epidemiology. The proximal humerus fractures are the third-leading fractures in humans. It occurs mostly in the elderly.

Therapy. There are operative and non-operative options for the treatment of those fractures. The treatment options are based upon degree of dislocation and morphology of the fracture. The long-term results of twoand three-part humerus head fractures treated with intramedullary nail or locking plates shows no difference. However the standard surgical treatment of four-part humerus head fractures, which could not be treated non-operatively, should be the locking plate. Avoiding complications. Regardless of the surgical procedure, both the anatomic reposition and good reconstruction of the calcar are essential to avoid postoperative complications.

\section{Keywords}

Humeral head · Bone nails · Bone plates . Anatomical reposition . Calcar reconstruction
2. lyengar JJ, Devcic Z, Sproul RC, Feeley BT (2011) Nonoperative treatment of proximal humerus fractures: a systematic review. J Orthop Trauma 25:612-617

3. Kim SH, Szabo RM, Marder RA (2012) Epidemiology of humerus fractures in the United States: nationwide emergency department sample, 2008. Arthritis Care Res (Hoboken) 64:407-414

4. Konrad G, Audigé L, Lambert S et al (2012) Similar outcomes for nail versus plate fixation of threepart proximal humeral fractures. Clin Orthop Relat Res 470:602-609

5. Krappinger D, Bizzotto N, Riedmann S et al (2011) Predicting failure after surgical fixation of proximal humerus fractures. Injury 42:1283-1288

6. Neer CS (1970) Displaced proximal humeral fractures. II. Treatment of three-part and four-part displacement. J Bone Joint Surg Am 52:1077-1089

7. Ockert B, Braunstein V, Kirchhoff C et al (2010) Monoaxial versus polyaxial screw insertion in angular stable plate fixation of proximal humeral fractures: radiographic analysis of a prospective randomized study. J Trauma 69:1545-1551
8. Olerud P, Ahrengart L, Ponzer S et al (2011) Internal fixation versus nonoperative treatment of displaced 3-part proximal humeral fractures in elderly patients: a randomized controlled trial. J Shoulder Elbow Surg 20:747-755

9. Priemel M, Domarus C von, Klatte TO et al (2010) Bone mineralization defects and vitamin $D$ deficiency: histomorphometric analysis of iliac crest bone biopsies and circulating 25-hydroxyvitamin D in 675 patients. J Bone Miner Res 25:305-312

10. Südkamp NP, Audigé L, Lambert S et al (2011) Path analysis of factors for functional outcome at one year in 463 proximal humeral fractures. J Shoulder Elbow Surg 20:1207-1216

11. Sun JC, Li YL, Ning GZ et al (2013) Treatment of three- and four-part proximal humeral fractures with locking proximal humerus plate. Eur J Orthop Surg Traumatol 23:699-704

12. Zhu Y, Lu Y, Shen J et al (2011) Locking intramedullary nails and locking plates in the treatment of two-part proximal humeral surgical neck fractures: a prospective randomized trial with a minimum of three years of follow-up. J Bone Joint Surg Am 93:159-168 